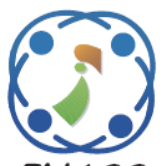

\title{
Performance Analysis of Systematic Raptor Codes in Low Power Regime
}

\author{
Dhafer R. Zaghar ${ }^{1} \quad$ Hadeel N. Abdullah ${ }^{2 *} \quad$ Ikhlas M. Farhan ${ }^{2}$ \\ ${ }^{1}$ Electrical Engineering Department, Al-Mustansiriyah University, Iraq \\ ${ }^{2}$ Electrical Engineering Department, University of Technology, Iraq \\ * Corresponding author's Email: 30002@uotechnology.edu.iq
}

\begin{abstract}
This paper investigates the application of advanced forward error correction techniques mainly: Raptor code, Low-Density Parity Checks (LDPC) code, and Luby Transform (LT) for wireless communication systems in the low power regime (LPR). Raptor codes are a significant theoretical and practical improvement over LT codes, which were the first actual class of fountain codes. These codes are under consideration for many recent technologies. Different code parameters such as code rate and several decoding iterations are used to show their effect on the performance of digital systems. In this paper, we propose a systematic Raptor code that enabled transmission of information with different code rates in LPR. Simulation results showed that the suggested design is appropriate for data transmission in a low SNR regime and can provide a reasonable bit error rate (BER) against the additive white Gaussian noise(AWGN) with the optimum selection rate1/4 and 1/9. A more significant improvement in the system's BER performance for the SNR up to $-5 \mathrm{~dB}$ over binary input additive white Gaussian noise (BI-AWGN) channels that showed through simulation results. Also, the BPSK modulation with systematic Raptor code is performed better than QPSK modulation in a limited power region, for the same coding rate and the number of decoding iterations.
\end{abstract}

Keywords: Raptor codes, Rateless codes, LT codes, LDPC, Low SNR, BIAWGN.

\section{Introduction}

The transmitted data over wireless channels affected because these channels will suffer from degradation due to noise and fading. Thus in the last decade, researchers focus on developing transmission for these channels were the main challenge in wireless applications such as Wireless Sensor Networks (WSN). The most important way to protect transmitted data is the collaboration between the sender and receiver during the communication; this can be done by using the Error Control Coding (ECC) techniques [1]. Forward Error Correction schemes (FEC) are the most potent useful error control schemes with much digital communication application.

Channel coding is a technique used in digital communication systems to detect and correct the errors which occurred in the transmitted signal during the transmission process. It improves the performance of various wireless applications, along with improving energy efficiency [1].

In a Low SNR (LSNR) environment, numerous communication systems like ad-hoc wireless networks, ultra-wideband, and wireless sensor networks operate for each node in the network, where the degrees of freedom available is excellent [2]. However, many wireless applications do not have access to such helpers to improve reliability like in cellular-based on machine-to-machine communications. Also, the large number of lowpower devices randomly transmitted their data to one base station. Many tools may be in deep fading due to their constant movement.

For this type of network, allocating resources, like the bandwidth or even relays, to devices, is ineffective because of the random nature of the transmission [3]. This type of network requires a study of effective transmission strategies in the LSNR regime, as it is not possible to increase the transmission power or to use cooperative 
technologies. Hence, channel coding is a successive option.

Fountain codes [4] are a class of erasure codes which have inherently rate-less and able to generate an infinite quantity of encoded bits symbol from the source symbols $\mathrm{k}$. This feature makes it suitable for application in cases where the rate of channel erasure is not known previously. D. MacKay in [5], shows the most important benefits that the fountain codes have in terms of efficiency, robustness, and reliability have attributed to the fixed code- rate within the fading and AWGN channels.

The first practical investigation of Fountain codes is the Luby Transform (LT) code [6, 7]. The LT codes that are invented by Luby and used to enhance the weight distribution for rateless codes. Where the degree of every coding symbol is acquired from one probability density function, indicated by the "degree distribution." The soliton distribution initially used as a grading distribution within LT codes. However, it showed that the capacity approaches of LT code have an average score increased moderately with the information size using a logarithmic slope; as a result, it is challenging for designing a coding system with a linear timing [2].

Many researchers strived for improving performance the LT coding with protected the information across wireless applications, where the packet erasures, noise, and fading are encounter. For the purpose of improvement, the error correction ability of the LT codes and the complexity of these charts tend to increase. The decoding process of the LT codes uses the probability decoded mechanism for low-density parity-check (LDPC) codes [8]. Hence, for improving the performance of LT codes in the antagonistic wireless channel, a systematic LT (SLT) code has been suggested in [9].

In [10], A. Shokrollahi has proposed the first class investigation of fountain codes called Raptor codes. They are an extended of the LT codes. While LT codes have nonlinear encoding and decoding costs [7] and suffer from relatively high error floors on noisy channels, Raptor codes overcome these issues by adding a linear block coding as a pre-code to encode source symbols before the inner LT encoder [10].

The pre-code may be a sequence of multiple codes usually, the LDPC code [8-11-12] coupled with another linear code such as Hamming code [13]. Due to their rate-less nature, fountain codes can be adapted to changes for channel condition that the block codes maybe it fails in the adaptation process.

Raptor codes collect the feature of block codes and fountain codes, to produce a new versions of fountain codes with linear encoding/decoding costs and the possibility of rate-less systems in adapting channel conditions. However, raptor codes can fully recovering the source of information with few overhead and linear coding time, these practices for many applications requiring a high data rate [14] .

When Raptor code is non-systematic, this feature is one of its drawbacks, which indicates that source symbols are not necessary to reproduce among output symbols. Therefore, the systematic Raptor codes provided an advantage of eliminating the decoding process when the packet is not lost in transmission. Systematic Raptor codes produced by using the SLT codes as the component codes [9].

However, the systematic Raptor code can be considered as a necessary code in many applications. For example, assuming that the Raptor code is deployed in phases through some receivers are equipped with the decoder and not others. Hence, if non-systematic Raptor codes are used for this application, then the sender needs to keep tracking of the various receivers and transmitting the uncoded message to receivers without a decoding process, and the coded message to the other ones.

Clearly, if a systematic Raptor code is used, the burden of administration is less for transmitting. Moreover, it is essential in a variety of other applications as the transmission of videos through the communication networks [10]

In this paper, systematic releases of Raptor codes will be presented and analyzed, and the main features in the present work are:

- An arrangement of FEC like systematic Raptor code is proposed to improve the performance of wireless applications in low power regime while trying to gain an advantage BER in low SNR.

- The proposed design focuses on the Binary Phase Shift Keying (BPSK) and Quadrature Phase Shift Keying (QPSK) modelation technique with the same rate for the LDPC and SLT codes in BI-AWGN channel, where the initial data of the log-likelihood ratio (LLR) is symmetric Gaussian distribution.

The remaining sections of the paper are organized as follows; Section 2 presents the essential relevant works, section 3 deals with the Encoding and Decoding of Raptor codes considered in work. Details of the proposed system include the Raptor codes model together with its parameters and specifications, also given in section 4. Section 5 presents the Simulation results. Finally, the main concluding remarks presented in section 6.

\section{Related work}


FEC codes are useful for protecting information message through added redundancy. The receiver can be corrected errors among the transmitted message with FEC codes. Recent works showed that with applied rate-less principles, the reliability of wireless transmission can be significantly improving. Unlikely conventional codes, rate-less codes that have adapted for differing channel conditions. Rateless code are a preferred coding technology for FEC at the application of multimedia broadcast/multicast service over cellular networks. There are two types of rateless codes: Luby transform (LT) codes and Raptor codes.

Many researchers have the interest to implement rateless codes into modern communication systems.

S. Kuo, Y. Guan, S. Lee, and M. Lin [15] proposed the design of Raptor codes in adaptive degree distribution in the BIAWGN to achieve code rates that approach the channel capacity associated with a broad range of SNR. While in [16] introduced the design of raptor code with a degree distribution in the erasure channels and noisy channels.

S. Chen, Z. Zhang, L. Zhang, and C. Yao [17]. Discuss the complexity of decoding Raptor code reduces by removing all edge associated with the high LLR values without improving the performance of the BER.

M. Shirvanimoghaddam and S. Johnson [2], suggested designing for Raptor codes using the practical degree distribution in the LSNR system. Also, the decoding complexity and the upper limit of the maximum coding rate efficiency derived from specific design parameters.

M. Shirvanimoghaddam, S. Johnson, and A. Lance [18] introduced Raptor codes to achieve very high efficiency in very LSNRs, which make it very engaging utilized in continuous-variable quantum key distribution (CV-QKD) systems.

A. Kharel and L.Cao [19] Introduced improved for the decoding process of Raptor code using GaussJordan elimination (GJE). The decoding process can continue to generate the non-zero value of LLR updated to each input symbol in the Tanner graph. Also, BER performs much better than a conventional technique at a broad range of SNR transmission overheads.

In [20], an analysis of raptor code channels by discretized density evolution (DDE) introduced in BIAWGN. The critical bit error rate from the optimization DDE approach suggested for providing optimum output with a degree distribution, which moreover reduction gap to Shannon boundaries. This method outperforms the traditional Raptor codes.

Z. Xu, C. Yang, Z. Tan and Z. Sheng [21] proposed a raptor code scheme in in-vehicle PLC systems that it is appropriate in in-vehicle data transmission and providing proper protect against noise environment. At the same time, a considerable reception overhead needed.

Y. Xu, G. Zhang, H. Ju, D. He, J. Sun, Y. Wu, and W. Zhang [14], suggested design of Raptor-like LDPC which reduction the decoding complexity, comparing with the conventional belief propagation (BP) decoding algorithm. Also, raptor-like LDPC code providing the optimization with first iterate number.

Previous researches have improved interest to study and implement Raptor code at various stages into digital communication systems. However, the study and analysis a degree distribution designing for Raptor codes in the LSNR system and a few limits of the maximum rate efficient of coded/decoded complexity have rarely been examined in previous research without discussing the relationship between SNR and BER in practical digital modulation and demodulation systems. To address this issue, this paper proposed a systematic Raptor code with digital modelation technique, like BPSK and QPSK .where the design enabled data transmission scheme for different code rates in LSNR regime.

\section{Raptor codes encoding and decoding}

The main structure of the raptor code consists of a combination of an outer high-rate LDPC and an inner SLT code. Raptor codes consist of two levels of encoding and decoding processes [20], as illustrated in Fig. 1. Firstly, the input symbols $\mathrm{s}, \mathrm{s}=\left[s_{1}, s_{2}, s_{3} \ldots\right.$, $\left.\left.s_{k}\right]\right)$, si $\epsilon\{0,1\}$ are pre-coded with LDPC code to produce $\mathrm{k}^{\prime}$ intermediates symbols $(\mathrm{u}), \mathrm{u}=\left[u_{1}, u_{2}, \ldots\right.$, $\left.u k^{\prime}\right], \mathrm{u}_{\mathrm{i}} \in\{0,1\}, \mathrm{k}^{\prime}>\mathrm{k}$.

Then, these intermediates symbols are encoded by inner SLT code to generate the output symbol c, where $\mathrm{c}=\left[c_{1}, c_{2}, \ldots, c_{n}\right], \mathrm{c}_{\mathrm{i}} \in\{0,1\}, \mathrm{n}>\mathrm{k}^{\prime}$. Thus, the Raptor code can achieve for any code-rate $(k / n)$ depends on the generated codes word length.

Also, we assume BPSK modulation that mapping $0 \rightarrow+1$ and $1 \rightarrow-1$. Given an AWGN channel with input $\mathrm{x}= \pm 1$, the received signal modeled can be described as yi $=\mathrm{x}_{\mathrm{i}}+\mathrm{n}_{\mathrm{i}}$, where $\mathrm{x}_{\mathrm{i}}=1-2 \mathrm{c}_{\mathrm{i}}$, which represents the modulated of code-word and $n_{i}$, is the zero-mean Gaussian noise. The LLR can be calculated for each received symbol as follows [19, 20]:

$$
\mathrm{L}(\mathrm{cj}) \quad=\ln \frac{p\left(c_{j}=0 / y_{j}\right)}{p\left(c_{j}=1 / y_{j}\right)}=\frac{2 y_{j}}{\sigma^{2}}
$$




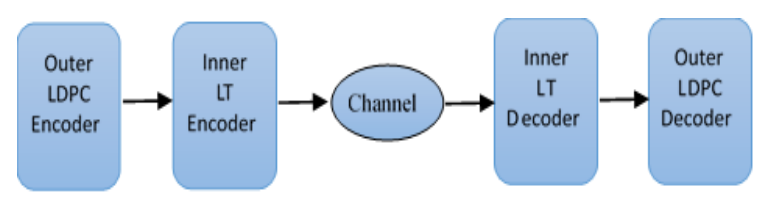

(a)

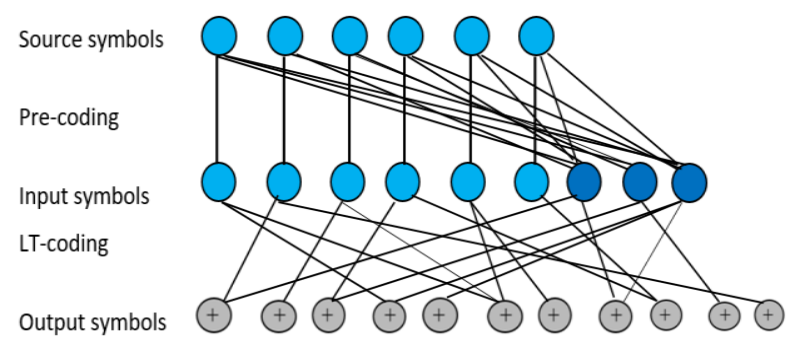

(b)

Figure. 1 Raptor codes: (a) Structure and (b) Tanner graph [19]

Where $\sigma^{2}$ is variance (power) of Gaussian noise, and $\mathrm{n}_{\mathrm{i}} \sim \mathrm{N}\left(0, \sigma^{2}\right)$ is the channel noise with mean zero and variance equal to $\sigma^{2}$.

In the decoding part, the two-step sequential decoding developed in [22] by decoding the inner system, followed by decoding outer code. For the inner decoder, the sum-product algorithm (SPA) is working through decoding a Tanner graph that generated utilize the intermediate as variable nodes (VNs) and output symbols as check nodes (CNs). The LLR updated from $j_{\text {th }} C N$ to $i_{\text {th }} V N$ referred to $\mathrm{L}_{\text {cjvi }}$ also from ith $\mathrm{VN}$ to jth $\mathrm{CN}$, which indicates that $\mathrm{L}_{\mathrm{vic} \text { j }}$ calculated as [22]:

$$
\begin{gathered}
L_{C_{j} V_{i}}=2 \tanh ^{-1}\left(\left(\tanh \frac{L\left(C_{j}\right)}{2}\right) \prod_{i^{\prime} \in N_{c}(j)-\{i\}} \tanh \left(\frac{L_{i^{\prime}} C_{j}}{2}\right)\right) \\
L_{V_{i} C_{j}}=L\left(V_{i}\right)+\sum_{j^{\prime} \in N_{v}}(j)-\{j\} \\
L_{C_{j} V_{i}}
\end{gathered}
$$

Where $\mathrm{Nc}(\mathrm{j})$ means the collection of VNs linked to the jth check node $(\mathrm{CN})$, also, Nv(i) shows the CNs set that connects to the ith VN. After accomplishing the inner decoding process, The final LLR is added with each other using decision rule $L(v i)+\sum j \in N v(i)$ Lc jvi for each variable node (VN), which utilized as channel estimated the outer decoder. Since the SPA used by the inner and outer decoding process of the Raptor code, Let the code rates of inner $\left(\mathrm{R}_{\mathrm{i}}\right)$ and outer codes $\left(\mathrm{R}_{\mathrm{o}}\right)$, where the outer code rate is $R o=K / K^{\prime}$, and the inner code rate is $R i=K^{\prime} / N$ so that the Raptor code has achieved code rate as [22]:

$$
R=R_{I} R_{O}
$$

Also, in [23], assume to transmit one codeword, the channel condition $\left(\mathrm{E}_{\mathrm{b}} / \mathrm{N}_{\mathrm{o}}\right)$, overall code-rate $(\mathrm{R})$, and noise quantity $(\sigma)$ related to:

$$
E_{b} / N_{o}=10 \log _{10}\left(1 /\left(2 R \sigma^{2}\right)\right)
$$

\section{Proposed system model}

The proposed system model for the physical layer architecture of the systematic Raptor codes system illustrated in Fig. 2. Assuming that Raptor code with parameters $(\mathrm{k}, \mathrm{C}, \Omega(\mathrm{x}))$, Where $\mathrm{C}$ Is the pre-code with message length $\mathrm{k}$ and block length $n$, and let $\Omega(\mathrm{x})$ a degree distribution of LT code which has a dependable decoding process of overhead $\epsilon$.

We design an encoding algorithm which accepted $\mathrm{k}$ input symbols $\mathrm{x}_{1}, \mathrm{x}_{2}, \ldots, \mathrm{x}_{\mathrm{k}}$ and produces a set of $\left\{\mathrm{i}_{1}\right.$, $\left.\mathrm{i}_{2}, \ldots, \mathrm{i}_{\mathrm{k}}\right\}$ with separate $\mathrm{k}$ Indexes from 1 to $\mathrm{k}(1+\varepsilon)$, and unlimited string $\mathrm{z}_{1}, \mathrm{z}_{2}, \ldots$ of output symbols such that $z_{i l}=x_{1}, \ldots, z_{i k}=x_{k}$, and such that the output symbols may be efficiently calculated. Thus, produced a group $\left\{i_{1}, i_{2}, \ldots, i_{k}\right\}$ indicated the systematic positions, and the related output symbols called as systematic output symbols, whereas the last remained output symbols are considered to be nonsystemic output symbols. So the general structure of the algorithm is as follows:

i- Calculation of systematic positions $i_{1}, i_{2}, \ldots, i_{k}$. Leads to irreversible matrix $R_{k \times k}$. This data is calculated via sampling the $\mathrm{k}(1+\varepsilon)$ times the $\Omega(\mathrm{x})$ separately for obtaining the vectors $\mathrm{v}_{1}$, $\mathrm{v}_{2}, \ldots, \mathrm{v}_{\mathrm{k}(1+\varepsilon)}$, and apply the decoding technique modification to these vectors.

ii- Matrix $R=A . G$ is the product of Matrix A, with generator matrix $G$. whereby the first $\mathrm{k}(1+\varepsilon)$ symbol are obtained using $\mathrm{v}_{1}, \ldots, \mathrm{v}_{\mathrm{k}(1+\varepsilon)}$

iii- Next, using $\mathrm{R}^{-1}$ to convert the encoding input symbols $\mathrm{x}_{1}, \mathrm{x}_{2}, \ldots, \mathrm{x}_{\mathrm{k}}$ to the intermediate symbols $\mathrm{u}_{1}, \mathrm{u}_{2}, \ldots, \mathrm{u}_{\mathrm{k}}$.

iv- Applying the Raptor codes to the intermediate symbols will produce the first $\mathrm{k}(1+\varepsilon)$ symbols Using pre-sampled vectors $\mathrm{v}_{1}, \mathrm{v}_{2}, \ldots, \mathrm{v}_{\mathrm{k}(1+\varepsilon)}$

v- For decode systematic Raptor code, the first step of the decoding process was applied to the origin Raptor code to get an intermediate symbols $\mathrm{u}_{1}$, $\mathrm{u}_{2}, \ldots, \mathrm{u}_{\mathrm{k}}$, and then using the matrix $\mathrm{R}$ to convert this intermediate symbols back to the original input symbols $\mathrm{x}_{1}, \mathrm{x}_{2}, \ldots, \mathrm{x}_{\mathrm{k}}$. 


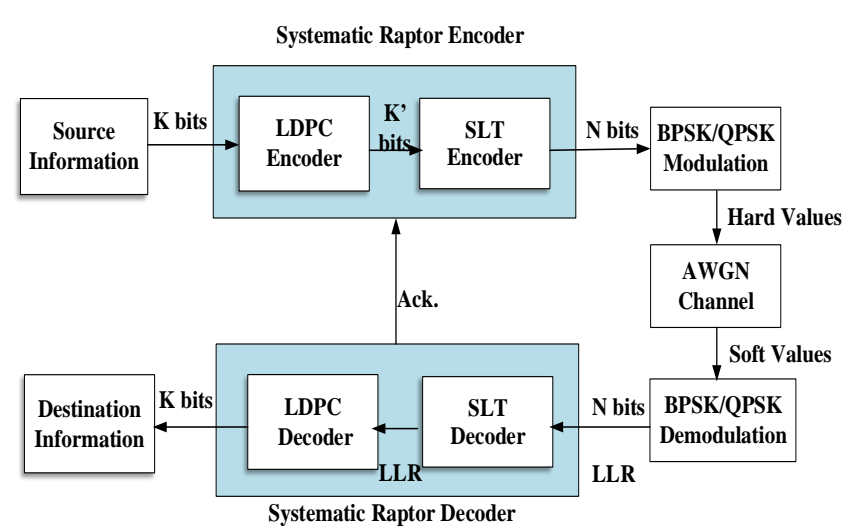

Figure. 2 Systematic raptor code system model

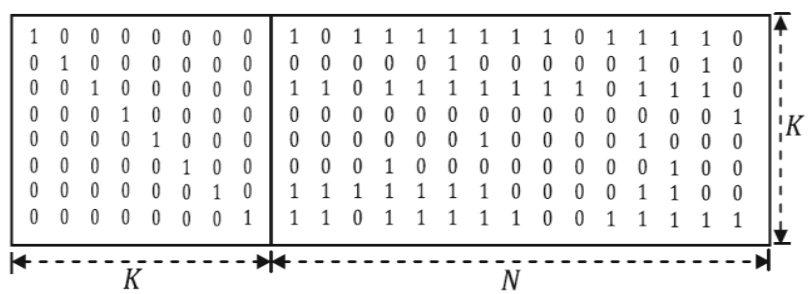

Figure. 3 The systematic LT generator matrix [8]

However, to express the matrix algebra, SLT codes, as shown in Fig. 3, have been suggested in the present work by expanding the LT codes $[\mathrm{K} \times \mathrm{N}]$ generator matrix by aiding a unity matrix with size $[\mathrm{K}$ $\mathrm{x} \mathrm{K}]$. The systematic LT generation matrix $\mathrm{G}$ is related to the LDPC parity check matrix $\mathrm{H}$ of as $\mathrm{G}_{\mathrm{K} \mathrm{xN}}=$ $\left[\mathrm{I}_{\mathrm{K} \times \mathrm{K}} / \mathrm{A}_{\mathrm{kx} \times \mathrm{M}}\right]$, where $\mathrm{A}$ is a non-singular matrix with the size of $[\mathrm{K} \times \mathrm{M}]$. Also, $\mathrm{N}=\mathrm{K}+\mathrm{M}$, which represents the number of columns in $\mathrm{G}$ and $\mathrm{K}$, represents a number of rows in $\mathrm{G}$.

However, the transmitter side includes three main blocks. The first block describes the source information. Second, the random information passes across an irregular LDPC encoder that produces the intermediate codes which are using by the SLT encoder to create the encoded symbols. Third, the Raptor encoder output mapped in modulation scheme, such as BPSK and QPSK, which considered in [2425]. However, to process input information, the transmitter system was beginning by an identical both the LDPC and SLT encoders.

In general, the longer length of the Raptor encoder gives better performance compared to the shortest. However, the larger Raptor code length means more sophisticated Raptor decoding in the system receiver.

Also, the ultimate goal for the receiving device is to be able to retrieve source data with minimal probability of error and with the possibility of channel imperfection. The receiver process begins with the demodulation process, which is the same modulation plan used in the transmitter of the system. Decoding process starting in the decoding SLT belief algorithm that applied to the LLR from the demodulated signal. This latter is processed by the outer LDPC decoder, based on the belief propagation algorithm. Finally, the performance of the coding process can be evaluated by BER measure.

\section{Simulation results}

For a digital communications system, the quality of the transmission can be described in term of the Bit Error Rate (BER) probability. The BER is used as an essential parameter in characterizing the performance of communication channels. BER is used as a necessary parameter in describing the performance of communication channels. Where, The BER is performed via compared the transmitting series of bits to the receiving bits and calculating the number of errors. Also, there is a relationship between SNR and BER that depends on the modulation scheme, coding, and a range of other factors in digital communications systems.

However, The BER vs. SNR is the leading performance measure considered for the digital communication system under the LSNR regime.

In the simulation, two different codes were used to construct Raptor codes: LDPC codes and SLT codes with varying rates of coding $(1 / 2$ and $1 / 3)$ and a different number of decoding iterations $(10,20$, and 30). Also, we simulated 1000 blocks with two different modulation technique BPSK and QPSK, over the AWGN channel model.

The BER performance across the BI-AWGN channel considered in the work shown in Figs. 4 - 11, and the remaining codewords results are shown in Tables 1 and 2.

Figs. 4 and 5 discuss the results listed in Table 1. Whereas the results obtained, show that Raptor codes with a rate of $1 / 2$ to LDPC / SLT codes for BPSK provided an improvement in SNR of about $3 \mathrm{~dB}$ compared to QPSK with the same rate, the equal length $\mathrm{N}$ of the codeword is 9600 bits and message length $\mathrm{K}$ is 4800 bits code and the corresponding decoding iterations of 10 at BER of $10^{-5}$ (Fig. 6).

The corresponding improvements are about 3.2 $\mathrm{dB}$ for low rate 1/9 raptor code with QPSK compared to the rate $1 / 4$ for the same message length $\mathrm{k}$ is 6000 bits code and decoding iterations 10 as shown in Fig. 7. Furthermore, we found that as shown in Figs. 4 - 7 show that with $\mathrm{R}=1 / 4 \mathrm{BER}$ in the range $10^{-3}$ at $0 \mathrm{~dB}$ and $-3 \mathrm{~dB}$ for QPSK and BPSK respectively, while the BER reaches $10^{-1}$ at $-3 \mathrm{~dB}$ and $-4 \mathrm{~dB}$ for QPSK and BPSk respectively. 
Fig. 8 shows BER performance in the system, where the performance of raptor code is better than that of LDPC code, and SLT code with the length N of a codeword is 192000 bits and information length $\mathrm{K}$ is 9600 bits code. Figs. 9 - 11 show BER performance of raptor code with code rate $1 / 9$ with
BPSK and QPSK, where the performance of raptor is better than rate $1 / 4$ with the same parameters. The general behavior of the codes here shows better performance of low rate codes compared to the high rate.

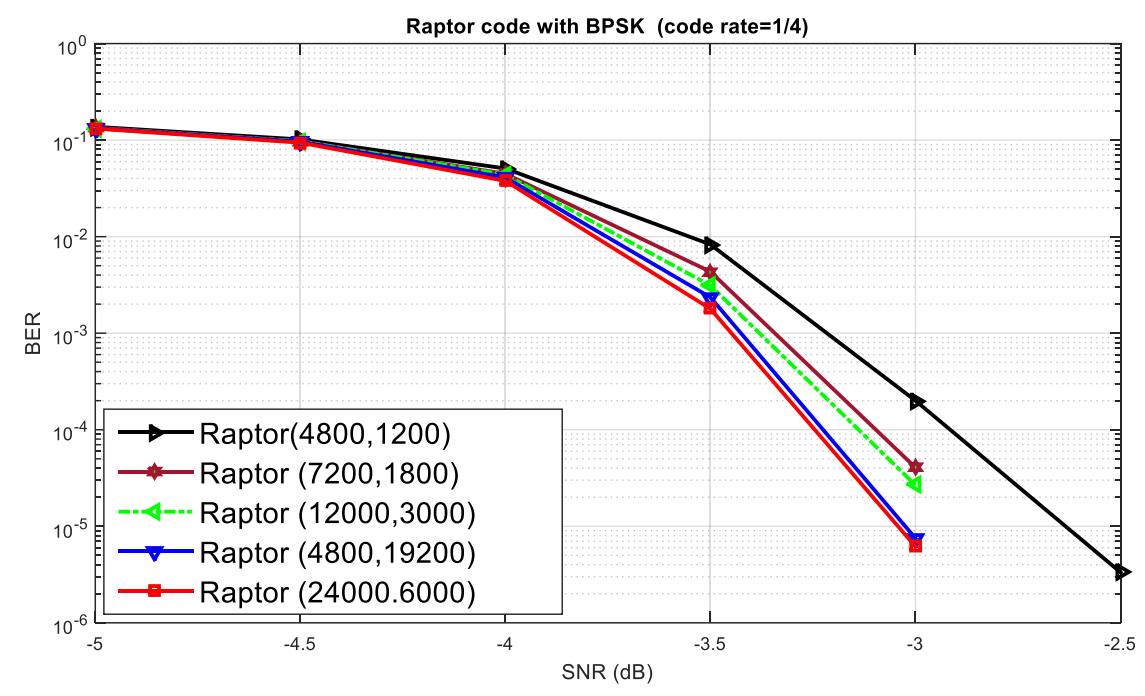

Figure. 4 Raptor codes ( $R=1 / 4$, BPSK)

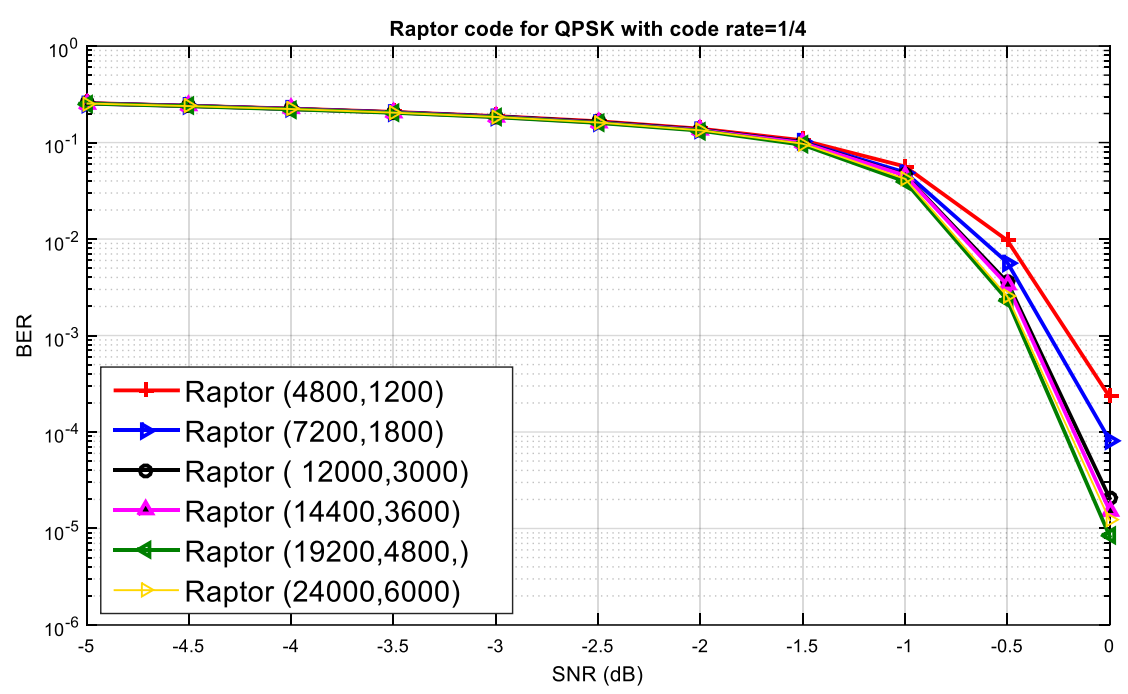

Figure. 5 Raptor codes (R=1/4, QPSK) 


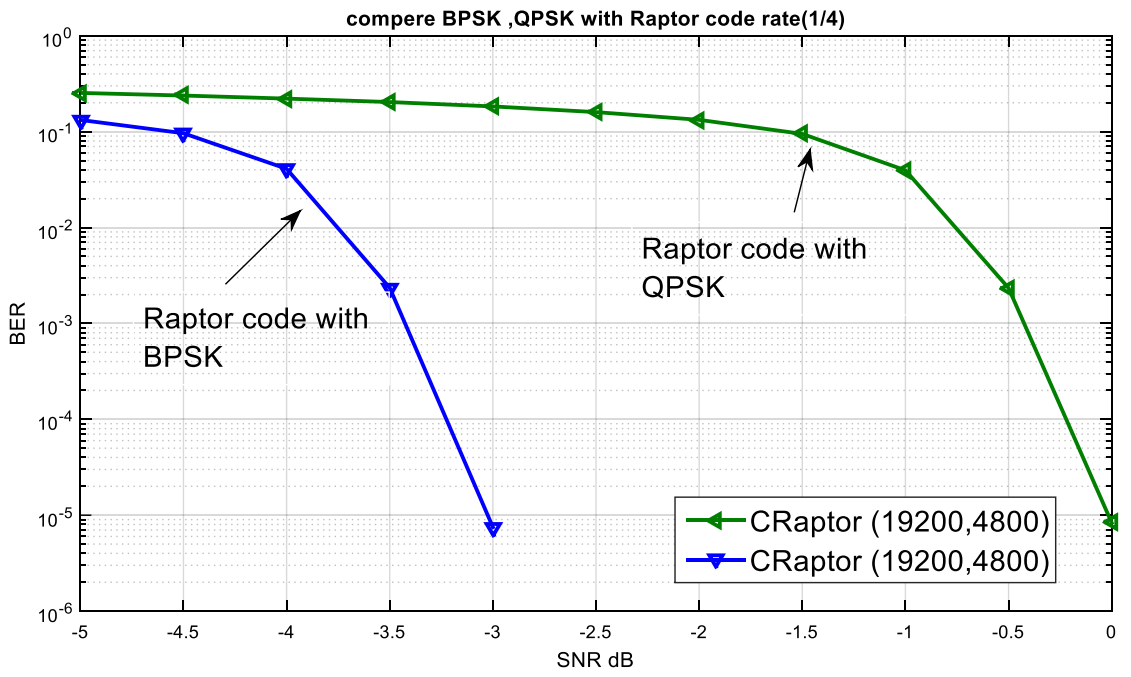

Figure. 6 Raptor codes ( $\mathrm{R}=1 / 4$, BPSK/QPSK)

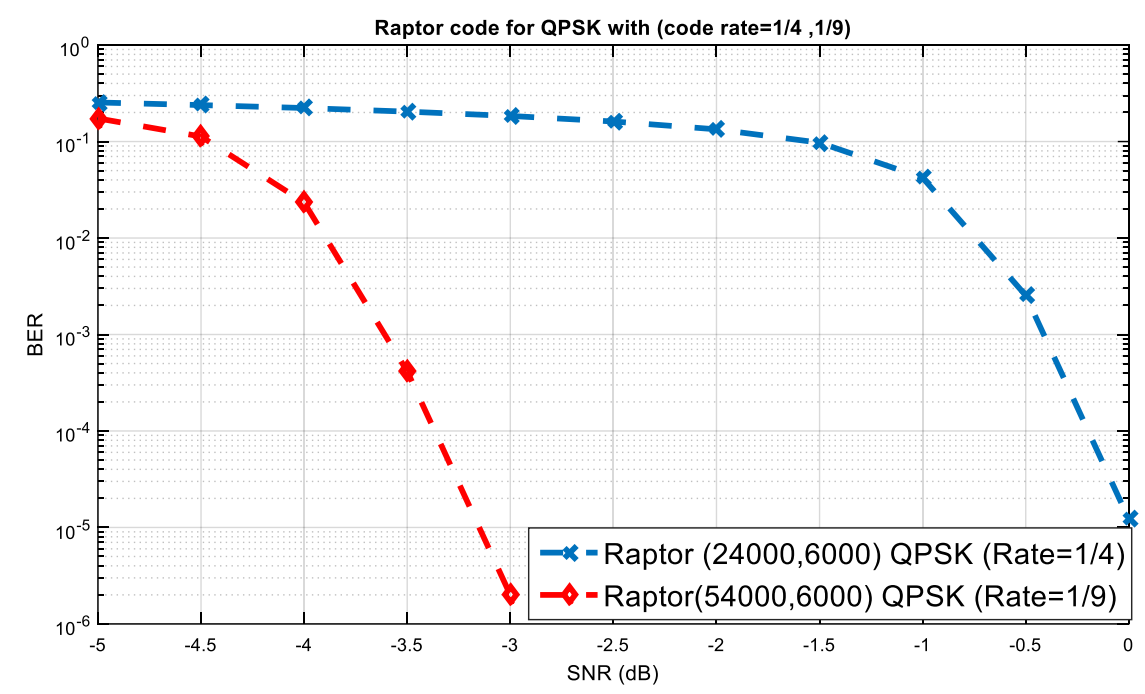

Figure. 7 Raptor codes ( $\mathrm{R}=1 / 4,1 / 9, \mathrm{QPSK})$

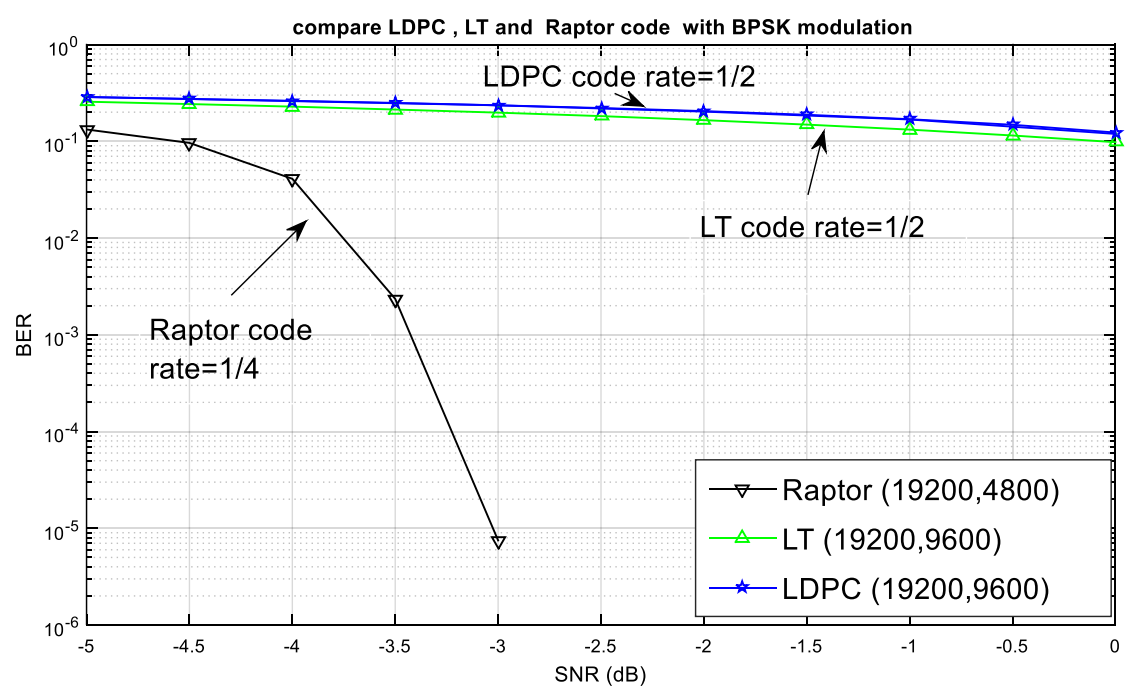

Figure. 8 Coding schemes with BPSK. 


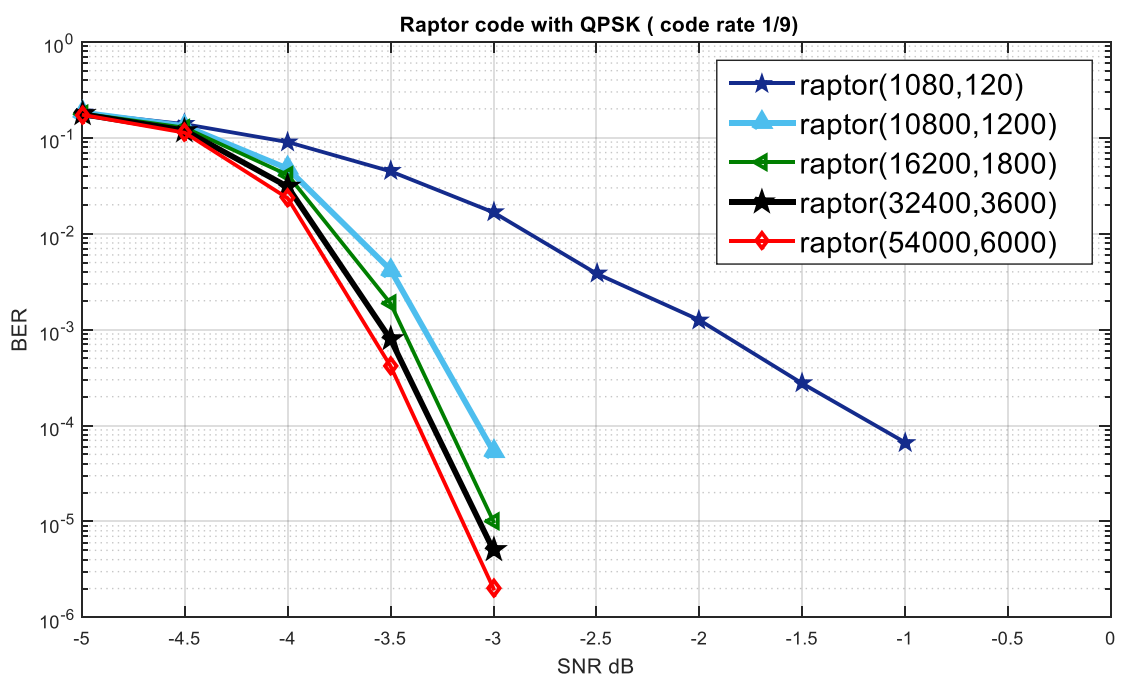

Figure. 9 Raptor $\operatorname{codes}(\mathrm{R}=1 / 9$, QPSK $)$

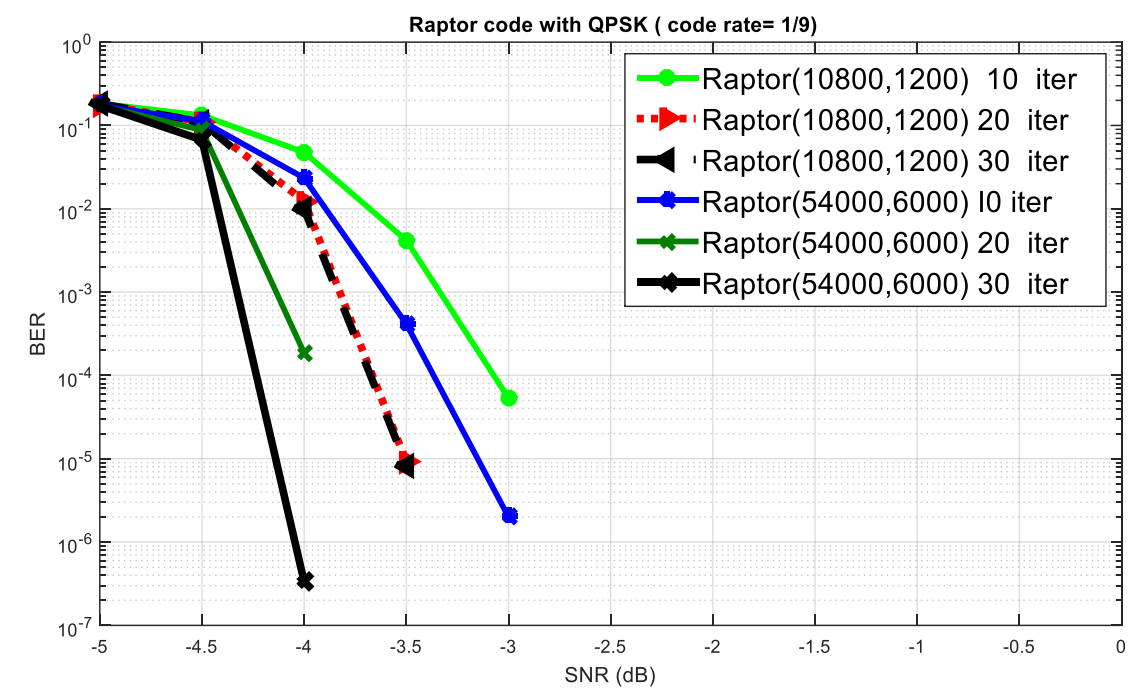

Figure. 10 Raptor $\operatorname{codes}(\mathrm{R}=1 / 9$, QPSK.10, $20 \& 30$ iterations)

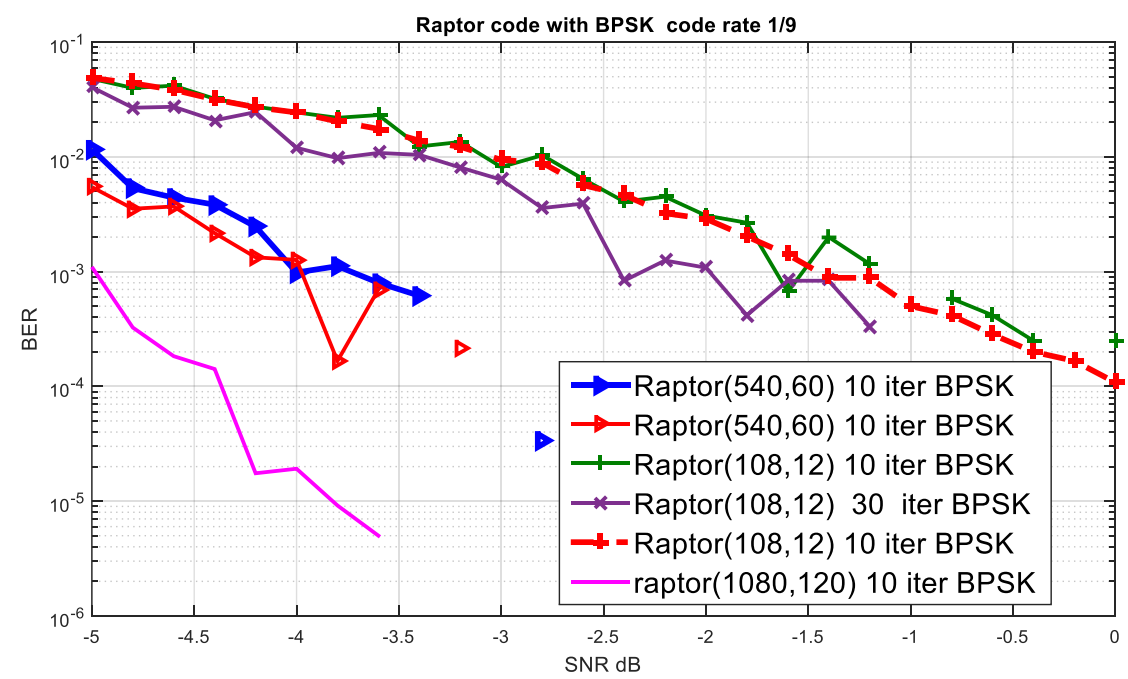

Figure. 11 Raptor codes ( $\mathrm{R}=1 / 9, \mathrm{BPSK}, 10,20 \& 30$ iterations) 
Table 1. Parameters of used coding schemes (Raptor code rate=1/4)

\begin{tabular}{|c|c|c|c|c|c|c|}
\hline NO. & $\begin{array}{c}\text { LDPC Code } \\
\text { Parameter } \\
(\mathbf{K}, \mathbf{K})\end{array}$ & $\begin{array}{c}\text { SLT Code } \\
\text { parameter } \\
\left(\mathbf{N}, \mathbf{K}^{\prime}\right)\end{array}$ & $\begin{array}{c}\text { LDPC/SLT } \\
\mathbf{C o d e} \text { Rate } \\
\left(\mathbf{K}^{\prime} / \mathbf{K}, \mathbf{K}^{\prime} / \mathbf{N}\right)\end{array}$ & $\begin{array}{c}\text { Raptor Code } \\
\text { parameter } \\
(\mathbf{N}, \mathbf{K})\end{array}$ & $\begin{array}{c}\text { Raptor } \\
\text { Code Rate } \\
(\mathbf{K} / \mathbf{N})\end{array}$ & $\begin{array}{c}\text { Number-of } \\
\text { iterations } \\
\text { in decoding }\end{array}$ \\
\hline 1 & $(2400,1200)$ & $(4800,2400)$ & $1 / 2$ & $(4800,1200)$ & $1 / 4$ & $10,20,30$ \\
\hline 2 & $(3600,1800)$ & $(7200,3600)$ & $1 / 2$ & $(7200,1800)$ & $1 / 4$ & $10,20,30$ \\
\hline 3 & $(6000,3000)$ & $(12000,6000)$ & $1 / 2$ & $(12000,3000)$ & $1 / 4$ & $10,20,30$ \\
\hline 4 & $(7200,3600)$ & $(14400,7200)$ & $1 / 2$ & $(14400,3600)$ & $1 / 4$ & $10,20,30$ \\
\hline 5 & $(9600,4800)$ & $(19200,9600)$ & $1 / 2$ & $(19200,4800)$ & $1 / 4$ & $10,20,30$ \\
\hline 6 & $(12000,6000)$ & $(24000,12000)$ & $1 / 2$ & $(24000,6000)$ & $1 / 4$ & $10,20,30$ \\
\hline
\end{tabular}

Table 2. Parameters of used coding schemes (Raptor code rate=1/9)

\begin{tabular}{|c|l|l|c|c|c|c|}
\hline NO. & $\begin{array}{c}\text { LDPC Code } \\
\text { Parameter } \\
\left(\mathbf{K}^{\prime}, \mathbf{K}\right)\end{array}$ & $\begin{array}{c}\text { SLT Code } \\
\text { parameter } \\
\left(\mathbf{N}, \mathbf{K}^{\prime}\right)\end{array}$ & $\begin{array}{c}\text { LDPC/SLT } \\
\mathbf{C o d e} \text { Rate } \\
\left(\mathbf{K}^{\prime} / \mathbf{K}, \mathbf{N} / \mathbf{K}^{\prime}\right)\end{array}$ & $\begin{array}{c}\text { Raptor Code } \\
\text { parameter } \\
(\mathbf{N}, \mathbf{K})\end{array}$ & $\begin{array}{c}\text { Raptor } \\
\text { Code Rate } \\
(\mathbf{K} / \mathbf{N})\end{array}$ & $\begin{array}{c}\text { Number-of } \\
\text { iterations } \\
\text { in decoding }\end{array}$ \\
\hline 1 & $(360,120)$ & $(1080,360)$ & $1 / 3$ & $(1080,120)$ & $1 / 9$ & $10,20,30$ \\
\hline 2 & $(3600,1200)$ & $(10800,3600)$ & $1 / 3$ & $(10800,1200)$ & $1 / 9$ & $10,20,30$ \\
\hline 3 & $(5400,1800)$ & $(16200,5400)$ & $1 / 3$ & $(16200,1800)$ & $1 / 9$ & $10,20,30$ \\
\hline 4 & $(10800,3600)$ & $(32400,10800)$ & $1 / 3$ & $(32400,3600)$ & $1 / 9$ & $10,20,30$ \\
\hline 5 & $(14400,4800)$ & $(43200,14400)$ & $1 / 3$ & $(43200,4800)$ & $1 / 9$ & $10,20,30$ \\
\hline 6 & $(18000,6000)$ & $(54000,18000)$ & $1 / 3$ & $(54000,6000)$ & $1 / 9$ & $10,20,30$ \\
\hline
\end{tabular}

\section{Conclusion}

This paper provides a practical, systematic Raptor code with modulation techniques such as BPSK and QPSK that enabled data transmission scheme for different code rates for BI_AWGN in the LSNR regime, wherever the forward error correction schemes can improving the performance of wireless communication systems in the LPR. The performance of the variable-rate of systematic Raptor codes with the performance of the two different rates of LDPC/SLT codes in terms of BER show in the simulation test results. Among the simulation results, there are two primary outcomes; the first result represents the Raptor code with the overall code rate fixed to $1 / 4$ shows that an improvement in the performance of the BER system with the increased length of the codeword, as shown in Figs. 4 - 6 .The second result obtained by using the Raptor codes for the code rate $1 / 9$ instead of the rate $1 / 4$, which shows a more significant improvement in the system's BER performance. It is essential to note that the result from simulation test shows that the proposed systematic Raptor over BIAWGN channels, which offer the BER of $10^{-6}$ at $\mathrm{SNR}=-4 \mathrm{~dB}$ with $(\mathrm{R}=1 / 9$, BPSK) with less decoding iterations of 10 compared to proposed work in [2], where the practical implementation of Raptor codes can achieve higher efficiency in LSNR regime when the maximum number of decoding iterations is 1000 .

\section{Conflicts of Interest}

The authors declare no conflict of interest.

\section{Author Contributions}

Conceptualization, H. Abdullah and D. Zaghar; methodology, D. Zaghar; software, I. Farhan; validation, I. Farhan, D. Zaghar, and H. Abdullah; formal analysis, I. Farhan; writing - original draft preparation, I. Farhan; writing - review and editing, H. Abdullah.

\section{References}

[1] M. S. Ali and A. A. Kadhim, "Coding Schemes for Energy Constrained IoT Devices", International Journal of Mobile Network Communications and Telematics (IJMNCT), Vol. 9, No. 2, 2019.

[2] M. Shirvanimoghaddam and S. Johnson, "Raptor codes in the low SNR regime", IEEE Transactions on Communications, Vol. 64, No. 11, pp. 4449-4460, 2016.

[3] M. Shirvanimoghaddam, Y. Li, M. Dohler, B. Vucetic, and S.Feng, "Probabilistic rateless multiple access for machine-to-machine communication", IEEE Transactions on Wireless Communications, Vol. 14, No. 12, pp. 6815-6826, 2015.

[4] F. Lázaro, G. Liva, E. Paolini, and G. Bauch, "Distributed LT codes with improved error floor performance", IEEE Access, pp. 8102-8110, 2019. 
[5] D. J. MacKay, "Fountain codes", IEE Proceedings-Communications, Vol. 152, No. 6, pp. 1062-1068, 2005.

[6] J. He, I. Hussain, Y. Li, M. Juntti, and T. Matsumoto, "Distributed LT codes with improved error floor performance", IEEE Access, 7, pp. 8102-8110, 2019.

[7] M. Luby, "LT codes", In: Proc. of the 43rd Annual IEEE Symposium on Foundations of Computer Science, Vancouver, BC, Canada, pp. 271-280, 2002.

[8] R. Gallager, "Low-density parity-check codes", IRE Transactions on Information Theory, Vol. 8, No. 1, pp. 21-28, Jan. 1962

[9] T. D. Nguyen, L. L. Yang, and L. Hanzo, "Systematic Luby transform codes and their soft decoding", In: Proc. of IEEE Workshop on Signal Processing Systems, Shanghai, China, pp. 67-72, 2007

[10] A. Shokrollahi, "Raptor Codes", IEEE Transactions on Information Theory, Vol. 52, No. 6, pp. 2551-2567, Jun. 2006.

[11] D. J. MacKay and R. M. Neal, "Near Shannon limit performance of low-density parity-check codes", Electronics Letters, Vol. 32, No. 18, pp.1645-1646, 1996.

[12] D. J. C. MacKay, "Good error-correcting codes based on very sparse matrices", IEEE Transactions on Information Theory, Vol. 45, No. 2, pp. 399-431, 1999.

[13] R. W. Hamming, "Error detecting and errorcorrecting codes", The Bell System Technical Journal, Vol. 29, No. 2, pp.147-160, 1950.

[14] Y. Xu, G. Zhang, H. Ju, D. He, J. Sun, Y. Wu, and W. Zhang, "A Low Complexity Decoding Scheme for Raptor-Like LDPC Codes", IEEE Transactions on Broadcasting, Vol. 65, No. 4, pp.770-776. 2019.

[15] S.-H. Kuo, Y. L. Guan, S.-K. Lee, and M.-C. Lin, "A design of physical layer Raptor codes for wide SNR ranges", IEEE Communications Letters, Vol. 18, No. 3, pp. 491-494, 2014.

[16] J. Sorensen, T. Koike-Akino, P. Orlik, J. Ostergaard, and P. Popovski, "Ripple Design of LT Codes for BIAWGN Channels", IEEE Transactions on Communications, Vol. 62, No. 2, pp. 434-441, 2014.

[17] S. Chen, Z. Zhang, L. Zhang, and C. Yao, "Belief propagation with gradual edge removal for Raptor codes over AWGN channel", IEEE International Symposium on Personal Indoor and Mobile Radio Communications, pp. 380385, 2013.

[18] M. Shirvanimoghaddam, S. J. Johnson, and A. M. Lance, "Design of Raptor codes in the low
SNR regime with applications in quantum key distribution", In: Proc. of IEEE International Conference on Communications (ICC), Kuala Lumpur, Malaysia pp. 1-6, 2016.

[19] A. Kharel and L. Cao, "Improved decoding for Raptor codes with short block-lengths over BIAWGN channel", In: Proc. of International Conference on Computer, Information and Telecommunication Systems (CITS), Kunming, China, pp. 1-5, 2016.

[20] A.Kharel and L.Cao, "Analysis and design of physical layer raptor codes", IEEE Communications Letters, Vol. 22, No. 3, pp. 450-453, 2017.

[21] Z. Xu, C. Yang, Z. Tan, and Z. Sheng, "Raptor code-enabled reliable data transmission for invehicle power line communication systems with impulsive noise", IEEE Communications Letters, Vol. 21, No. 10, pp. 2154-2157, 2017

[22] O. Etesami and A. Shokrollahi, "Raptor codes on binary memoryless symmetric channels", IEEE Transactions on Information Theory, Vol. 52, No. 5, pp. 2033-2051, 2006.

[23] S. Y. Chung, G. D. Forney, T. J. Richardson, and R. Urbanke, "On the design of low-density parity-check codes within $0.0045 \mathrm{~dB}$ of the Shannon limit", IEEE Communications Letters, Vol. 5, No. 2, pp. 58-60, 2001.

[24] F. Knabe, M. Wiese, C. Huppert, and J. Klotz, "The Wideband Slope Region of BPSK and QPSK for Broadcast Channels in the LowPower Regime", IEEE Information Theory Workshop, pp. 440-444, 2009.

[25] N. S. Alagha, "Cramer-Rao bounds of SNR estimates for BPSK and QPSK modulated signals", IEEE Communications Letters, Vol. 5, No. 1, pp. 10-12. 2001. 